\title{
La privación social y afectiva de la madre se asocia a alteraciones anatómicas y funcionales en el feto y recién nacido
}

\author{
Percy Pacora ${ }^{1,2,3}$, Elena Capcha ${ }^{3,4}$, Laura Esquivel ${ }^{3}$, Máximo Ayala ${ }^{1,2,3}$, \\ Wilfredo Ingar ${ }^{3,5}$, Lilia Huiza ${ }^{1,3,6}$
}

Resumen

Palabras clave

\begin{abstract}
Objetivo: Demostrar que la privación social y afectiva en la madre se asocia a restricción del crecimiento fetal, muerte fetal, parto pretérmino, alteraciones anatómicas y funcionales en el feto y recién nacido. Materiales y Métodos: Estudio retrospectivo en el que se analizó la base de datos materno-perinatal del Hospital San Bartolomé. Se examinó tres grupos de gestantes con privación social y afectiva: 1) madres solteras $(n=6561)$, 2) gestantes adolescentes con ausencia de control prenatal $(n=2131)$ y 3) primigestas con talla menor de $160 \mathrm{~cm}(n=35722)$. Se comparó las complicaciones maternas y perinatales con las gestantes : 1) madres con unión estable $(n=40629), 2)$ gestantes adolescentes con control prenatal $(n=7644)$ y 3$)$ primigestas con talla mayor de $159 \mathrm{~cm}(n=5264)$. Se empleó el odds ratio (OR) y el intervalo de confianza al 95\% (IC). Resultados: Las madres solteras presentaron mayor riesgo de falla en la función placentaria (OR 1,92, IC95\% 1,51-2,44), restricción del crecimiento fetal (OR 2,02, IC95\% 1,45-2,82), rotura prematura de membranas fetales (OR 1,28, IC95\% 1,00-1,63) comparadas con las madres con unión de pareja estable. Las gestantes adolescentes con ausencia de control prenatal presentaron mayor riesgo de muerte fetal (OR 2,89, CI95\% 1,85-4,50), rotura prematura de membranas fetales (OR 1,38, CI95\% 1,18-1,62), parto pretérmino (OR 1,63, CI95\% 1,391,91), neonato pequeño para la edad (OR 1,25, IC95\% 1,08-1,44), morbilidad neonatal (OR 1,23, IC95\% 1,07-1,41), asfixia perinatal (OR 2,73, IC95\% 1,33-5,58), neonato con Ápgar bajo en minuto 1 (OR 1,26, IC95\% 1,05-1,52), dificultad respiratoria (OR 1,51, IC95\% 1,102,06), sepsis neonatal (OR 1,54, IC95\% 1,19-1,99), prematuridad (OR 1,50, IC95\% 1,271,78), muerte neonatal (OR 2,56, IC95\% 1,88-3,48) y muerte perinatal (OR 3,02, IC95\% 2,244,07) comparadas con las gestantes adolescentes con control prenatal. Las primigestas con talla menor de $160 \mathrm{~cm}$ presentaron mayor riesgo de ausencia de control prenatal (OR 1,22, IC95\% 1,13-1,32), parto pretérmino (OR 1,12, IC95\% 1,00-1,26), desproporción fetopélvica (OR 1,49, IC95\% 1,11-2,02), parto por cesárea (OR 1,28, IC95\% 1,19-1,38), Ápgar bajo en minuto 1 (OR 1,20, IC95\% 1,07-1,35), recién nacido pequeño para la edad (OR 1,29, IC95\% 1,17-1,42) y recién nacido prematuro (OR 1,12, IC95\% 1,00-1,26) comparadas con la primigesta con talla mayor de $159 \mathrm{~cm}$. Conclusiones: Las mujeres con falta de apoyo social y afectivo, como son las madres solteras, las mujeres adolescentes sin cuidado prenatal y las mujeres de talla baja, presentan riesgo aumentado en la salud materna y perinatal. Estos resultados sugieren que la privación social y/o afectiva en la madre repercute negativamente en el resultado perinatal, generando alteraciones anatómicas y funcionales en el feto y recién nacido.
\end{abstract}

Depresión; ansiedad; pobreza; carencia psicosocial; embarazo; feto; recién nacido; asfixia neonatal.
1 Instituto de Patología. Facultad de Medicina, Universidad Nacional Mayor de San Marcos (UNMSM). Lima, Perú.

2 Departamento de Gineco-Obstetricia. Facultad de Medicina, UNMSM. Lima, Perú.

3 Hospital Nacional Docente Madre-Niño San Bartolomé. Lima, Perú.
4 EscuelaAcadémico Profesional de Enfermería. Facultad de Medicina, UNMSM. Lima, Perú.

5 Departamento de Pediatría. Facultad de Medicina Universidad Nacional Mayor de San Marcos (UNMSM). Lima, Perú.

6 Departamento de Anatomía-Patológica. Facultad de Medicina, UNFV. Lima, Perú. 
Social and emotional deprivation in the mother is associated with anatomical and functional alterations in the fetus and newborn

\section{Abstract}

Objective: To ascertain that social and emotional deprivation of the mother is associated with fetal growth restriction, fetal death, preterm birth and congenital defects. Materials and Methods: We retrieved the maternal and perinatal outcome from the Informatic Perinatal System of all pregnancies with antenatal care whose delivery occurred between January 1, 1992 and December 31, 2001. Three groups of patients with social and emotional deprivation were included: 1) single women $(n=1285), 2)$ teenage women with absent prenatal care (APC), $(n=2131)$, and 3) primigravid with stature lower than $160 \mathrm{~cm}(n=35722)$. Maternal and perinatal outcome were compared with: 1) women living with sexual partner $(n=5550), 2)$ teenage women with prenatal care $(n=7644)$, and 3) primigravids taller than $159 \mathrm{~cm}(n=5264)$. Odds ratio and 95\% confidence interval (CI) were calculated. Results: Single women presented greater risk of placental failure (OR 1,92, 95\%CI 1,51-2,44), fetal growth restriction (OR 2,02, 95\%CI 1,45-2,82), and premature rupture of fetal membrane (OR 1,28, 95\%CI 1,00-1,63) as compared with women living with sexual partners. Teenagers presented greater risk of fetal death (OR 2,89, 95\% CI 1,854,50 ), premature rupture of fetal membranes (OR 1,38, 95\% CI 1,18-1,62), preterm labor (OR 1,63, 95\% CI 1,39$1,91)$, small for gestational age infant (OR 1,25, 95\%CI 1,08-1,44), neonatal morbidity (OR 1,23, IC95\% 1,071,41 ), perinatal asphyxia (OR 2,73, 95\%CI 1,33-5,58), low one minute Apgar score (OR 1,26, 95\%CI 1,05-1,52), respiratory distress syndrome (OR 1,51, 95\%CI 1,10-2,06), neonatal sepsis (OR 1,54, 95\%CI 1,19-1,99), prematurity (OR 1,50, 95\%CI 1,27-1,78), neonatal death (OR 2,56, $95 \% C I 1,88-3,48)$, and perinatal death (OR 3,02, 95\%CI 2,24-4,07) as compared with teen-age women without absent prenatal care. Primigravids shorter than $160 \mathrm{~cm}$ presented greater risk of absent prenatal care (OR 1,22, 95\%CI 1,131,32), preterm labor (OR 1,12, 95\%CI 1,00-1,26), fetopelvic disproportion (OR 1,49, 95\%CI 1,11-2,02), cesarean section delivery (OR 1,28, 95\%CI 1,19-1,38), low one minute Apgar score (OR 1,20, 95\%CI 1,07-1,35), small for gestational age infant (OR 1,29, 95\%CI 1,17-1,42), and preterm neonate (OR 1,12, 95\%CI 1,00-1,26) as compared to primigravids with taller than $159 \mathrm{~cm}$. Conclusions: Women with social and/or emotional privation, such as single pregnant women, teenage pregnant women with no prenatal care and women with low stature, are at increased perinatal risk of anatomical and functional alterations in the fetus and newborn.

Keywords: Depression; anxiety; poverty; psychosocial deprivation; pregnancy; fetus; infant, newborn; asphyxia neonatorum.

\section{INTRODUCCIÓN}

La enfermedad del ser humano antes de nacer (embrión-feto) se manifiesta clínicamente por restricción del crecimiento fetal, muerte fetal, nacimiento prematuro, alteraciones anatómicas y funcionales de diferentes sistemas del organismo $\left({ }^{1-8}\right)$. Se estima que $60 \%$ de los embarazos en el año 2000 en el Perú no fueron deseados y $35 \%$ de ellos terminaron en abortos inducidos $\left({ }^{9}\right)$. Además, la mayor parte de los embarazos no deseados por los padres que no fallecen antes de las 20 semanas de gestación presentan mayor riesgo de enfermedades en la vida fetal, neonatal, infantil y alteraciones en el aprendizaje y la conducta social del individuo adulto $\left({ }^{10-12}\right)$. Es por ello que, se asume que la mayor parte de la teratogénesis humana es producida por la conducta del mismo hombre $\left({ }^{13,14}\right)$.

Por otro lado, se reconoce internacionalmente que el nivel socio-económico bajo $\left(^{15,16}\right)$, el grado bajo de instrucción $\left({ }^{17-19}\right)$, la soltería $\left({ }^{20,21}\right)$, la ausencia de control prenatal $\left({ }^{22,23}\right)$ y la desnutrición materna $\left({ }^{24-26}\right)$ son factores de riesgo importantes en el embarazo. Este grupo particular de mujeres tiene en común una gran falta de ayuda social y de afecto, factores que afectan la salud de la madre y el recién nacido $\left.{ }^{(27}\right)$. El objetivo de nuestra investigación fue demostrar que la privación social y afectiva de la madre se asocia a restricción del crecimiento fetal, muerte fetal, parto pretérmino, alteraciones anatómicas y funcionales en el feto y recién nacido de un grupo de gestantes en Lima.

\section{MATERIALES Y MÉTODOS}

Se realizó un estudio analítico de una cohorte retrospectiva de gestantes cuyos partos fueron atendidos en el Hospital Nacional Docente Madre-Niño San Bartolomé, en Lima, desde el 1 de enero 1992 al 31 de diciembre 1999. De la base de datos del sistema automatizado perinatal del Hospital San Bartolomé se obtuvo el resultado materno y perinatal de tres grupos de 
mujeres que fueron considerados en estado de privación social y afectiva: 1) solteras, 2) adolescentes sin cuidado prenatal y 3) primigestas con talla menor de $160 \mathrm{~cm}$.

Las complicaciones maternas y perinatales de las mujeres consideradas con privación social y afectiva fueron comparadas con la contraparte de tres grupos de mujeres consideradas que no presentaban estado de privación social y afectiva: 1) mujeres en unión estable, 2) adolescentes con cuidado prenatal y 3 ) primigestas con talla mayor de $159 \mathrm{~cm}$. Se empleó el odds ratio (OR) y el intervalo de confianza al 95\% (IC95\%) para el análisis estadístico de los resultados.

Se consideró complicación materna a la presencia de una o más complicaciones médicas asociadas al embarazo (complicación médica) o directamente relacionadas al embarazo (complicación obstétrica).

Se denominó complicación perinatal a las enfermedades de la madre y el recién nacido relacionados al proceso del nacimiento, hasta los 28 días de producido éste.

Se llamó cuidado prenatal a la presencia de por lo menos una atención de la gestante durante la gestación a cargo de un médico, una obstetriz o enfermera.

\section{RESULTADOS}

El grupo 1 comprendió 47190 gestantes, $13,9 \%$ (6561/47190) fue soltera y 86,1\% (40629/ 47190) tuvo pareja estable (casada/conviviente). La Tabla 1 muestra el riesgo materno y perinatal de las mujeres solteras comparadas con las que tenían unión estables. Las mujeres solteras tuvieron en forma significativa mayor riesgo de complicaciones maternas y perinatales, tales como, complicaciones maternas, anemia crónica, preeclampsia, restricción del crecimiento fetal, rotura prematura de membranas, infección puerperal, neonato pequeño para la edad de gestación, morbilidad neonatal y trastorno metabólico neonatal (hipoglicemia/ hipocalcemia).
Tabla 1. Riesgos de la salud materna y perinatal de mujeres solteras comparadas con mujeres en unión estable.

\begin{tabular}{lcc}
\hline Complicaciones & OR & IC 95\% \\
\hline Infección puerperal & 1,52 & $1,25-1,84$ \\
Complicación materna & 1,28 & $1,22-1,35$ \\
Anemia crónica & 1,08 & $1,01-1,15$ \\
Restricción del crecimiento fetal & 1,47 & $1,26-1,71$ \\
Recién nacido PEG & 1,31 & $1,19-1,44$ \\
Trastorno metabólico neonatal & 1,23 & $1,03-1,47$ \\
Morbilidad neonatal & 1,22 & $1,12-1,32$ \\
Rotura prematura de membranas & 1,20 & $1,11-1,31$ \\
Preeclampsia & 1,16 & $1,07-1,24$ \\
\hline
\end{tabular}

OR: odds ratio; IC 95\%: intervalo de confianza al 95\%. PEG: Pequeño para la edad de gestación.

El grupo 2 comprendió 9775 gestantes, 21,8\% (2131/9775) no tuvo cuidado prenatal y $79,2 \%$ (7644/9775) tuvo cuidado prenatal. La Tabla 2 muestra el riesgo materno y perinatal de las mujeres adolescentes que no tuvieron cuidado prenatal comparado con el de las mujeres adolescentes con cuidado prenatal. Las adolescentes sin cuidado prenatal presentaron significativo mayor riesgo de muerte fetal, rotura prematura de membranas, parto pretérmino, endometritis puerperal, hemorragia puerperal, parto pretérmino, recién nacido pequeño para la edad de gestación, morbilidad neonatal, síndrome hipóxico-isquémico (asfixia) neonatal, neonato con Ápgar bajo en minuto 1, dificultad respiratoria, sepsis neonatal, prematuridad, muerte neonatal y muerte perinatal.

El grupo 3 comprendió 40986 primigestas, $12,8 \%(5264 / 40986)$ presentaba talla mayor de $159 \mathrm{~cm}$ y $87,2 \%(35722 / 40986)$ talla menor de $160 \mathrm{~cm}$. La Tabla 3 muestra el riesgo materno y perinatal de las mujeres primigestas con talla menor de $160 \mathrm{~cm}$ comparado con el de las mujeres primigestas mayores de $159 \mathrm{~cm}$. Las primigestas con talla menor de $160 \mathrm{~cm}$ presentaron significativo mayor riesgo de ausencia de cuidado prenatal, desproporción fetopélvica, parto por cesárea, Ápgar bajo en minuto 1, recién nacido pequeño para la edad y recién nacido prematuro. Las mujeres con talla menor de $160 \mathrm{~cm}$ 
Tabla 2. Riesgos de la salud materna y perinatal de mujeres adolescentes sin cuidado prenatal comparadas con mujeres adolescentes con cuidado prenatal.

\begin{tabular}{lcc}
\hline Complicaciones & OR & IC 95\% \\
\hline Hemorragia puerperal & 2,09 & $1,39-3,13$ \\
Muerte perinatal & 3,02 & $2,24-4,07$ \\
Muerte fetal & 2,89 & $1,85-4,50$ \\
Muerte neonatal & 2,56 & $1,88-3,48$ \\
Hipoxia e isquemia cerebral neonatal & 2,73 & $1,33-5,58$ \\
Parto prematuro & 1,63 & $1,39-1,91$ \\
Sepsis neonatal & 1,54 & $1,19-1,99$ \\
Dificultad respiratoria neonatal & 1,51 & $1,10-2,06$ \\
Rotura prematura de membranas & 1,38 & $1,18-1,62$ \\
Prematuridad neonatal & 1,50 & $1,27-1,78$ \\
Ápgar bajo en minuto 1 & 1,26 & $1,05-1,52$ \\
Recién nacido PEG & 1,25 & $1,08-1,44$ \\
Morbilidad neonatal & 1,23 & $1,07-1,41$ \\
Empleo de anticonceptivo posparto & 0,82 & $0,72-0,93$ \\
\hline
\end{tabular}

OR : Odds ratio ; IC 95\%: Intervalo de confianza al 95\%. PEG: Pequeño para la edad de gestación.

presentaron, en forma significativa, menor riesgo de preeclampsia-eclampsia (OR 0,88, IC95\% 0,82-0,95), infección urinaria (OR 0,89, IC95\% 0,82-0,97), parto vaginal espontáneo (OR 0,78, IC95\% $0,73-0,84)$ y recién nacido grande para la edad (OR 0,80, IC95\% 0,71-0,89) comparadas con las mujeres con talla mayor de $159 \mathrm{~cm}$.

\section{DISCUSIÓN}

El grupo estudiado en la presente investigación constituye un grupo seleccionado de mujeres embarazadas en Lima que decidieron continuar con la gestación. Este estudio consideró tres grupos de mujeres gestantes que se encontraban en situación especial de falta de afecto y de ayuda social, como: 1) las madres solteras que no cuentan con el afecto y la ayuda cercana del padre progenitor del hijo que lleva dentro de su organismo; 2) las gestantes adolescentes sin cuidado prenatal quienes se encuentran en particular situación de desventaja social y familiar; y, 3) las mujeres de talla menor de 160 $\mathrm{cm}$. Este último grupo de mujeres fue incluida en el estudio debido a que en el análisis de riesgo materno demostraron en forma significativa que presentaron mayor probabilidad de ausencia de cuidado prenatal (Tabla 3). La ausencia de control prenatal no sólo comprende la ausencia de atención profesional, sino que además es la consecuencia de: 1) la falta de un entorno social favorable que le permita a la mujer gestante acudir en busca de ayuda profesional; 2) la falta de fé de la mujer y su familia hacia los profesionales de la salud; y, 3) la falta de una actitud mental positiva y activa hacia el cuidado de su propia salud y la de su hijo $\left({ }^{27,28}\right)$. Además, se reconoce que la desnutrición crónica, material y afectiva durante la infancia se manifiesta con una talla baja en la edad adulta $\left({ }^{29-33}\right)$.

Encontramos que estos tres grupos de pacientes presentaron significativos riesgos en la salud de la madre, el feto y el recién nacido. El grupo con más graves consecuencias para la salud del hijo fue el grupo de madres adolescentes con ausencia de cuidado prenatal, ya que en este grupo de pacientes ocurrieron los riesgos de muerte fetal, neonatal y perinatal más significativos. En Suecia, Stephansson y colaboradores han demostrado que el nivel socioeconómico bajo aumenta el riesgo de muerte fetal, independiente de la edad materna, la talla, el índice de masa corporal, el hábito de fumar y el lugar de nacimiento $\left({ }^{32}\right)$. Nuestro estudio corrobora este hecho, ya que hemos encontrado que son las mujeres con mayor falta de ayuda social -las mujeres adolescentes sin cuidado prenatal- las que presentan riesgo significativo de muerte fetal y perinatal.

Tabla 3. Riesgos de la salud materna y perinatal de mujeres con talla menor de $160 \mathrm{~cm}$ comparadas con mujeres con talla mayor de $159 \mathrm{~cm}$.

\begin{tabular}{lcc}
\hline Complicaciones & OR & IC 95\% \\
\hline Ausencia de cuidado prenatal & 1,22 & $1,13-1,32$ \\
Desproporción feto-pélvica & 1,49 & $1,11-2,02$ \\
Recién nacido PEG & 1,29 & $1,17-1,42$ \\
Parto por cesárea & 1,28 & $1,19-1,38$ \\
Prematuridad neonatal & 1,12 & $1,00-1,26$ \\
Ápgar bajo en minuto 1 & 1,20 & $1,07-1,35$ \\
\hline
\end{tabular}

OR: Odds ratio; IC 95\%: Intervalo de confianza al 95\%.

PEG: Pequeño para la edad de gestación. 
El apoyo social y familiar en este grupo de pacientes parece ser un elemento fundamental, ya que estas mujeres después del parto tuvieron menos posibilidad de iniciar un método anticonceptivo que las adolescentes que tuvieron cuidado prenatal (Tabla 2).

En 1955, se reportó que las primíparas en Aberdeen, Escocia, que aumentaron en escala social al momento del matrimonio, fueron más altas, más sanas y tuvieron menos tasas de muerte perinatal $\left.{ }^{33}\right)$. La mujeres con falta de ayuda social (privación social) nacen de mujeres con deventaja social y la mejora de la salud materna y perinatal dependerá de la eliminación de la continuidad de esta desventaja social. La falta de apoyo social trae consigo la desnutrición física de la persona humana, la privación material, el aumento de los eventos negativos en la vida, la falta de afecto, el escaso nivel de educación y la falta de acceso a la información. De manera que, las mujeres con falta de ayuda social presentan un incremento en los problemas nutricionales y emocionales, incluyendo el estrés, la ansiedad, la depresión emocional y la baja autoestima, al igual que deficiencias cognitivas $\left({ }^{30}\right)$.

La salud es un estado de equilibrio entre el ser humano y su medio ambiente $\left({ }^{34}\right)$, De manera que, la salud depende de muchos factores además de los estrictamente médicos y/o biológicos. La participación activa de la familia y la comunidad es el componente más efectivo para la protección de la salud de la mujer y su hijo, debido a que $60 \%$ de las muertes fetales y neonatales en nuestro medio están asociados a un factor de privación social $\left({ }^{9,27}\right)$. El afecto, la solidaridad, la educación y las vías de acceso -cultural, social y geográfica- a los centros de atención médica son factores importantes para garantizar la salud de la madre y su hijo.

Si bien una limitación de nuestro estudio es la información obtenida en forma retrospectiva, donde no se investigó específicamente trastornos del ánimo en las gestantes, nosotros asumimos que los tres grupos de pacientes estudiados en esta investigación se encuentran en estado de privación social. Esta situación de privación social colocaría a estas mujeres en riesgo de sufrir trastornos del ánimo (depresión y/o ansiedad) durante el embarazo. La salud mental de la paciente y su entorno psicosocial probablemente sea más importante que la vigilancia biomédica de la gestante durante el embarazo para el pronóstico perinatal e infantil. Así, por ejemplo, la depresión emocional en el embarazo $\left({ }^{35}\right)$ incrementa el riesgo a la analgesia epidural durante el parto (RR 2,56, IC95\% 1,245,30 ), los nacimientos por cesárea (RR 2,28, IC $95 \% 1,15-4,53)$ y el ingreso del recién nacido a la unidad de cuidados neonatales (RR 2,18, IC95\% 1,02-4,66). Estos efectos permanecen siendo estadísticamente significativos aún cuando se controla para variables confusoras, tales como las complicaciones obstétricas.

La ansiedad antes del parto o el estrés materno se asocian con restricción del crecimiento fetal, el parto pretérmino, y analgesia epidural. Todas estas variables, al igual que el estrés y la falta de apoyo social, han sido identificadas como factores de riesgo de presentar un recién nacido con peso bajo al nacer $\left({ }^{35}\right)$.

Una revisión de 14 estudios, de los cuales 8 fueron metaanálisis, encontró que las mujeres que reportaron maltrato físico, emocional o abuso sexual durante el embarazo tenían riesgo incrementado en $40 \%$ de tener un recién nacido con peso bajo al nacer, comparadas con la mujer que no había sido víctima de maltrato (OR 1,4, IC95\% 1,1-1,8) $\left.{ }^{36}\right)$. Además, los hijos de mujeres deprimidas en el posparto presentan puntajes menores de cociente intelectual en forma significativa, problemas de atención y dificultades en el razonamiento matemático y mayor probabilidad de necesitar educación especial a la edad de 11 años que otros niños $\left({ }^{37}\right)$.

Por lo tanto, la alteración de la salud mental de la madre constituye el factor más importante que repercute negativamente en el bienestar del feto, el recién nacido y el infante, independiente de los cuidado médicos. Una madre, una familia o una sociedad, con depresión emocional no puede cuidar y estimular positivamente el 
desarrollo neurológico, cognitivo e intelectual del niño.

Es muy probable que las complicaciones obstétricas de origen 'idiopático' tengan como mecanismo fisiopatológico la privación social y emocional $\left.{ }^{38}\right)$. El trastorno del ánimo desencadena en el organismo materno la liberación de sustancias vasoactivas, el cortisol y la corticosterona. El exceso de cortisol en la sangre materna pasa al feto a través de la placenta y detiene el crecimiento fetal. Se ha demostrado, además, que los glicocorticoides en exceso son teratógenos en animales de experimentación y en humanos. La teratogénesis humana se manifiesta por restricción del crecimiento fetal, prematuridad, alteraciones anatómicas y funcionales de los órganos en la vida postnatal $\left({ }^{39-41}\right)$. Por otro lado, la placenta humana presenta la 11-beta hidroxiesteroide deshidrogenasa (11BHSD) tipo 2, que transforma el exceso de cortisol y corticosterona materno en sus metabolitos inactivos cortisona y 11dehidrocosticosterona $\left({ }^{42}\right)$. De manera, que la 11-BHSD tipo 2 representa una barrera al efecto nocivo del exceso de cortisol. La sobreexposición fetal a glicocorticoides endógenos maternos representaría el eslabón bioquímico que une el medio ambiente materno, con el crecimiento fetal y determinaría el tipo de desarrollo en el área neuroendocrina y en la esfera afectiva en la edad adulta. Por otro lado, se ha encontrado que la epinefrina y la norepinefrina suprimen la producción de 11BHSD tipo 2 en las células trofoblásticas humanas, lo que es mediado vía receptores adrenérgicos alfa $\left({ }^{43}\right)$. Además, se ha encontrado que las mujeres expuestas a estrés emocional negativo intenso tuvieron mayor frecuencia de hijos con anomalías de la cresta neural craneal (ej. defectos cráneo-faciales, sistema nervioso central, tracto de salida del corazón). El mayor riesgo se observó cuando estos eventos ocurrieron durante el primer trimestre de la gestación, debido a la muerte de un hijo mayor, especialmente si la muerte era inesperada $\left({ }^{44}\right)$.

Toda esta información nos señala la importancia de considerar la enfermedad y la muerte materna, fetal y neonatal de causa multifactorial donde la privación social y/o afectiva también participa $\left({ }^{27,45}\right)$.

En conclusión, este estudio demuestra que las mujeres con falta de apoyo social y afectivo, como son las madres solteras, las mujeres adolescentes sin cuidado prenatal y las mujeres de talla baja, presentan aumentados riesgos en la salud materna y perinatal.

La privación social y/o afectiva repercute negativamente sobre la salud materna y perinatal, tal como aparece en la Figura 1, generando alteraciones anatómicas y funcionales en el feto y recién nacido $\mathrm{y}$, eventualmente, teratogénesis en la descendencia.

\section{REFERENCIAS BIBLIOGRÁFICAS}

1. Pacora P, Romero R. El parto prematuro y el recién nacido prematuro. En: Pacheco J, ed. Ginecología y Obstetricia. $2^{\text {da }}$ Edicion. Lima (Peru): MAD Corp SA; 2006.

2. van Wassernaer A. Neurodevelopmental consequences of being born SGA. Pediatr Endocrinol Rev. 2005;2(3):3727.

3. Beckam DA, Brent RL. Mechanism of teratogenesis. Ann Rev Pharmacol Toxicol. 1984;24:483-500.

4. Mcmillen C, Robinson JS. Developmental Origins of the Metabolic Syndrome: Prediction, Plasticity, and Programming. Physiol Rev. 2005;85:571-633.

5. Kajantie E, Osmond C, Barker DJ, Forsen T, Phillips DI, Eriksson JG. Size at birth as a predictor of mortality in adulthood: a follow-up of 350000 person-years. Int $\mathrm{J}$ Epidemiol. 2005;34(3):655-63.

6. Barker DJ, Eriksson JG, Forsen T, Osmond C. Infant growth and income 50 years later. Arch Dis Child. 2005;90:272-3.

7. Barker DJ.The developmental origins of adult disease. J Am Coll Nutr. 2004;23(6 Suppl 1):588S-595S.

8. Painter RC, Roseboom TJ, van Montfrans GA, Bossuyt PM, Krediet RT, Osmond C, et al. Microalbuminuria in adults after prenatal exposure to the Dutch famine. J Am Soc Nephrol. 2005;16(1):189-94.

9. Ferrando D. El aborto inducido en el Perú: Hechos y cifras. Lima (Perú): Centro de la Mujer Flora Trisan, Pathfinder Internacional Fundacion Ford; 2002. Disponible en: http:// www.abortolegal.org/aborInducPeru.doc

10. David HP, Dytrych Z, Matejcek Z. Born unwanted. Observations from the Prague Study. Am Psychol. 2003;58(3):224-9.

11. David HP, Dytrych Z, Matejcek Z, Kubicka L, Roth Z, Dytrych $Z$, et al. The mental health of adults born of 


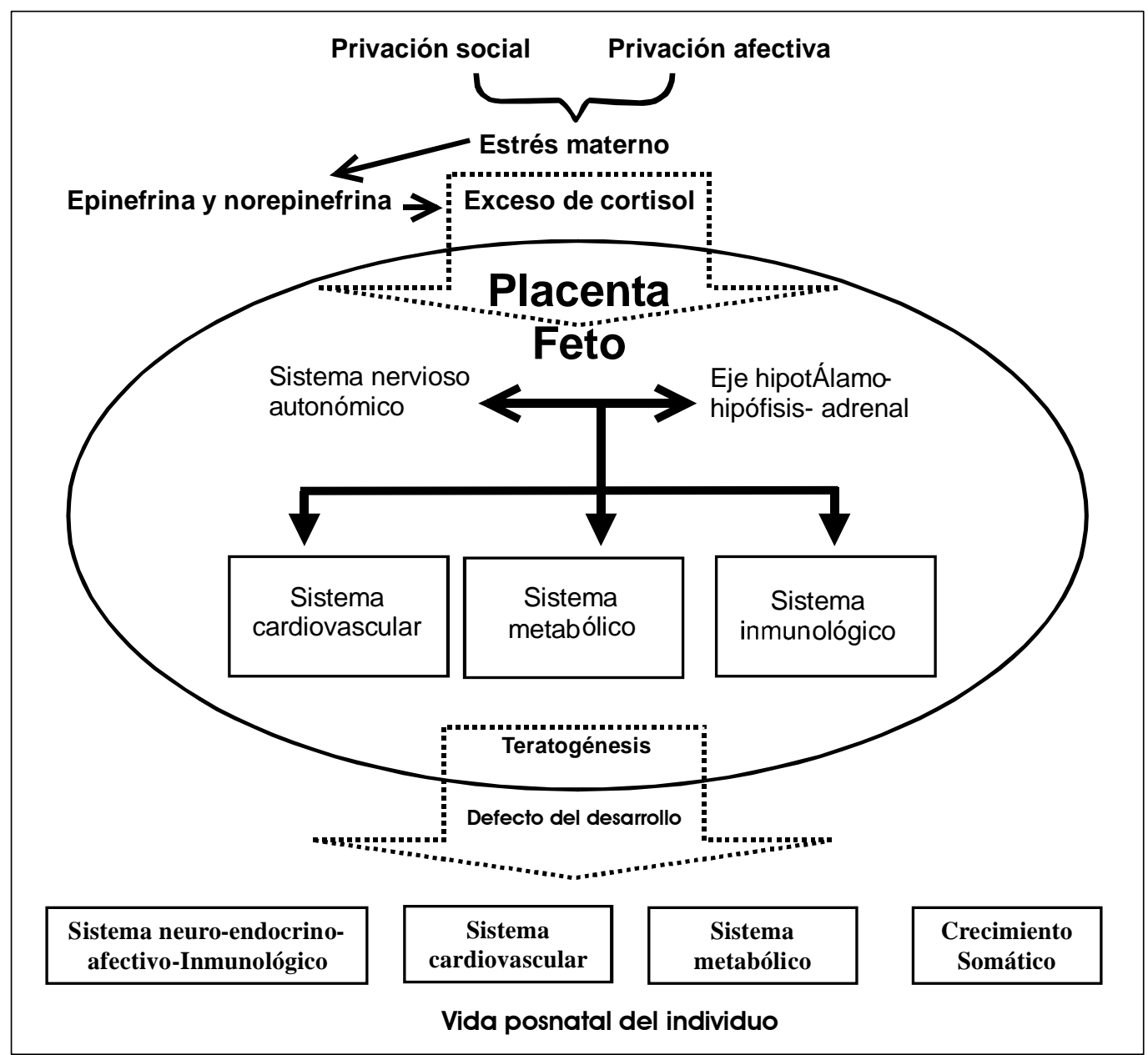

Figura 1. La privación social y afectiva de la madre genera teratogénesis en el hijo. El abandono social y afectivo intenso en la madre repercute en su eje hipotálamo-hipófisis-suprarrenal, desencadenando mayor concentración de cortisol y hormonas simpaticomiméticas. El exceso de cortisol materno supera la capacidad de la enzima placentaria humana 11-beta hidroxiesteroide deshidrogenasa (11-BHSD) tipo 2, la cual transforma el exceso de cortisol y corticosterona materno en sus metabolitos inactivos cortisona y 11-dehidrocosticosterona. La sobreexposición fetal a glicocorticoides endógenos maternos le expone a detención en el crecimiento fetal, alteraciones en el área neuroendocrina y en la esfera afectiva, el sistema cardiovascular y el sistema inmunológico del feto humano. Si el feto nace vivo, el ser humano presentará defectos en la función de estos sistemas en la vida posnatal. Las hormonas vasoactivas epinefrina y la norepinefrina tambien suprimen la producción de 11-BHSD tipo 2 en las células trofoblásticas humanas, agravando el efecto de los corticoides en el feto.

unwanted pregnancies, their siblings, and matched controls: a 35-year follow-up study from Prague, Czech Republic. J Nerv Ment Dis. 2002;190(10):653-62.

12. Del Campo C. Abortion denied-outcome of mothers and babies. Can Med Assoc J. 1984;131(6):546, 548.

13. Sood B, Delaney-Black V, Covington C, Nordstrom-Klee B, Ager J, Templin T, et al. Prenatal alcohol exposure and childhood behavior at age 6 to 7 years: I. dose-response effect. Pediatrics. 2001;108(2):E34.

14. Melnyk BM, Alpert-Gillis L, Feinstein NF, Crean HF, Johnson J, Fairbanks E, et al. Creating opportunities for parent empowerment: program effects on the mental health/ coping outcomes of critically ill young children and their mothers. Pediatrics. 2004;113(6): e597-607.

15. Stanley FJ, Hobbs MS. Perinatal outcome in Western Australia, 1968 to 1975: 2. Social and biological determinants. Med J Aust. 1981;1(8):414-6.

16. Read A, Stanley F. Postneonatal mortality in Western Australia 1970-1978. Aust Paediatr J. 1983;19(1):18-22.

17. de Sanjose S, Roman E. Low birthweight, preterm, and small for gestational age babies in Scotland, 1981-1984. J Epidemiol Community Health. 1991;45(3):207-10. 
18. Terry PB, Condie RG, Settatree RS. Analysis of ethnic differences in perinatal statistics. $\mathrm{Br}$ Med $\mathrm{J}$. 1980;281(6251):1307-8.

19. Ibrahim SA, Babiker AG, Amin IK, Omer MI, Rushwan $\mathrm{H}$. Factors associated with high risk of perinatal and neonatal mortality: an interim report on a prospective communitybased study in rural Sudan. Paediatr Perinat Epidemiol. 1994;8(2):193-204.

20. Bhoopalam PS, Watkinson M. Babies born before arrival at hospital. Br J Obstet Gynaecol. 1991;98(1):57-64.

21. Rantakallio P, Oja H. Perinatal risk for infants of unmarried mothers over a period of 20 years. Early Hum Dev. 1990;22(3):157-69.

22. Lumb KM, Congdon PJ, Lealman GT. A comparative review of Asian and British-born maternity patients in Bradford, 1974-8. J Epidemiol Community Health. 1981;35(2):106-9.

23. Menezes AM, Barros FC, Victora CG, Tomasi E, Halpern $\mathrm{R}$, Oliveira AL. Risk factors for perinatal mortality in Pelotas, a southern city of Brazil, 1993. Rev Saude Publica. 1998;32(3):209-16.

24. Steward JF, Stanley FJ. Comparison of births to Aboriginal and Caucasian mothers in Western Australia. Med J Aust. 1981;2(2):80-4.

25. Mavalankar DV, Trivedi CC, Gray RH. Maternal weight, height and risk of poor pregnancy outcome in Ahmedabad, India. Indian Pediatr. 1994;31(10):1205-12.

26. Agarwal DK, Agarwal KN, Satya K, Agarwal S. Weight gain during pregnancy-a key factor in perinatal and infant mortality. Indian Pediatr. 1998;35(8):733-43.

27. Huiza L, Pacora P, Ayala M y Villanueva M. La muerte fetal y neonatal es de origen multifactorial. An Fac Med Lima. 2003;64;1:13-20.

28. Pacora P. Prevención de la morbilidad y mortalidad en el Perú. En: Acción Científica, ed. Emergencias Obstétricas y Neonatales. Lima, Perú: Instituto Materno Perinatal; 2002. p.75-82.

29. Rao S, Joshi S, Kanade A. Growth in some physical dimensions in relation to adolescent growth spurt among rural Indian children. Ann Hum Biol. 2000;27(2):127-38.

30. Fernald LC, Grantham-McGregor SM. Stress response in school-age children who have been growth retarded since early childhood. Am J Clin Nutr. 1998;68(3):691-8.

31. Pacora P, Huiza L, Maymon E. Maternal stature lower than $160 \mathrm{~cm}$ identifies $90 \%$ of cases of maternal and perinatal mortality/morbidity in Lima, Peru. Am J Obstet Gynecol. 2002;187(6):S143. Abst.307.

32. Stephansson O, Dickman PW, Johansson ALV, Cnattingius $\mathrm{S}$. The influence of socioeconomic status on stillbirth risk. Int J Epidemiol. 2001;30:1296-1301.

33. Illsley R. Social class selection and class difference in relation to stillbirth and infant deaths. Br Med J. $1955 ; 11: 1520-4$
34. Delgado H. El Médico, la Medicina y el Alma. $3^{\text {ra }}$ Ed. Lima: UPCH Fondo editorial; 1992. p. 25-36.

35. Chung TK, Lau TK, Yip AS, Chiu HF, Lee DT. Antepartum depressive symptomatology is associated with adverse obstetric and neonatal outcomes. Psychosom Med. 2001;63(5):830-4.

36. Murphy CC, Berit S, Terri LM, Du Mont J. Abuse: A risk factor for low birth weight? A systematic review and metaanalysis. CMAJ. 2001;164(11):1567-72.

37. Hay DF, Pawlby S, Sharp D, Asten P, Mills A, Kumar R. Intellectual problems shown by 11-year-old children whose mothers had postnatal depression. J Child Psychol Psychiatry. 2001;42(7):871-89.

38. Peacock J1. Preterm delivery: effects of socioeconomic factors, psychological stress, smoking, alcohol, and caffeine. BMJ. 1995;311:531-5.

39. Robert E, Vollset SE, Botto L, Lancaster PAL, Merlob P, Cocchi G, et al. Malformation surveillance and maternal drug exposure: the MADRE project. Int J Risk Safety Med. 1994;6:75-118.

40. Czeizel AE, Rockenbauer M. Population-based case-control study of teratogenic potential of corticosteroids. Teratology. 1997; 56: 335-40

41. Rodríguez-Pinilla E, Martinez-Frias ML. Corticosteroids during pregnancy and oral clefts: a case-control study. Teratology. 1998;58:2-5.

42. Sakar S, Tsai S-W, Nguyen TT, Plevyak M, Padbury JF, Rubin LP. Inhibition of placental 11-hydroxysteroid dehydrogenase type 2 by catecholamines via-adrenergic signaling. APJ. 2001;281(6):R1966-74.

43. Welberg LA, Seckl JR, Holmes MC. Inhibition of 11betahydroxysteroid dehydrogenase, the foeto-placental barrier to maternal glucocorticoids, permanently programs amygdala GR mRNA expression and anxiety-like behaviour in the offspring. Eur J Neurosci. 2000;12(3):1047-54.

44. Hansen D, Lou HC, Olsen J. Serious life events and congenital malformations: a national study with complete follow- up. Lancet. 2000;356:875-80.

45. Huiza L, Pacora P, Reyes M, Ingar W, Santivañez A, Ayala M. La enfermedad perinatal/prematuridad es un sindrome clínico multifactorial: Asociación entre la herencia, la flora microbiana vaginal, el estado nutricional y la morbilidad perinatal. An Fac Medicina Lima. 2003;64(3):167-9.

Manuscrito recibido el 01 de julio de 2005 y aceptado para publicación el 30 setiembre de 2005.

Correspondencia: Dr. Percy Pacora Portella

Departamento de Gineco-Obstetricia - UNMSM

Hospital Nacional Docente Madre-Niño San Bartolomé

Av. Alfonso Ugarte 825. Lima 1, Perú

Correo-e:kalebna@terra.com.pe 